\title{
FLORE ALGALE DU RHIN CANALISÉ : DONNÉES RÉCENTES.
}

\section{J.F. PIERRE}

Hydrobiologie-Algologie, Université Henri Poincaré - Nancy I, Centre de 1er Cycle, B.P. 239, 54506 Vandoeuvre Cedex, France.

\section{RÉSUMÉ}

La communauté algale de trois stations du Rhin canalisé (Grand canal d'Alsace), au niveau du Centre de Production Nucléaire de Fessenheim, fait l'objet d'un suivi depuis 1977. Les présents résultats concernent la période 1988-1995. Ils mettent en évidence une stabilité de la flore algale au niveau de la diversité spécifique et de la répartition des espèces dominantes. L'inventaire diatomique s'enrichit d'une trentaine de taxons inédits pour ces stations. Ces données récentes confirment, au niveau de la communauté algale, l'absence d'impact visible du C.P.N. de Fessenheim.

Mots-clés : algues, diatomées, Rhin, site nucléaire.

\section{SURVEY OF THE ALGAL FLORA OF RHINE CANAL : RECENT DATA.}

\begin{abstract}
The algal community of canalized Rhine (Grand canal d'Alsace) has been surveyed since 1977. The present study concerns the period 1988-1995. Samples were taken from three localities, on each side, as well as on the actual site of the nuclear plant power, at Fessenheim (Haut-Rhin, France). The present results display the stability of the algal flora, both in specific diversity and repartition of the most abundant species. About thirty species are new for these stations. These recent data do not indicate any modification liable to be directly connected to the operation of nuclear plant power at Fessenheim.
\end{abstract}

Key-words : algae, diatoms, Rhine, nuclear plant power.

\section{INTRODUCTION}

II est rare que la communauté algale d'une station fasse l'objet d'un suivi sur plusieurs années. De ce point de vue, les stations du Rhin canalisé (Grand canal d'Alsace) au niveau du Centre de Production Nucléaire de Fessenheim offrent un intérêt exceptionnel car soumises, depuis 1977, à un contrôle algologique trimestriel. II devient possible, sur une aussi longue période, de tendre vers un relevé exhaustif des Algues du site et de dégager les caractéristiques des populations algales. Le présent travail complète une série de résultats déjà publiés (PIERRE, 1989a, 1989b).

Trois stations désignées par A, B et $C$ sont situées respectivement en amont du site électronucléaire, sur le site au niveau de l'entrée de la prise d'eau de refroidissement des condenseurs, et à l'aval du rejet de l'eau échauffée, immédiatement avant le barrage de la centrale hydraulique de Fessenheim (PIERRE, 1989a). 
Les prélèvements trimestriels (février ou mars, mai, août et septembre-octobre) sont réalisés par un personnel non algologue. Un raclage plus ou moins brutal sur une surface variable de la paroi bétonnée produit une importante quantité de sédiments organiques et minéraux. Le matériel prélevé est observé à l'état frais, puis traité pour l'étude des Diatomées.

\section{CARACTÉRISTIQUES PHYSIQUES ET CHIMIQUES}

L'impact du centre électronucléaire sur les caractéristiques hydrologiques moyennes des eaux du Rhin canalisé est apparu très limité. En particulier, les variations de température entre l'amont et l'aval sont de faible amplitude : de 0,4 à $1,7^{\circ} \mathrm{C}$.

La teneur en oxygène dissous est supérieure à $7 \mathrm{mg.l-1}$, donc proche de la saturation avec tendance à la sursaturation.

La conductivité est inférieure ou voisine de $550 \mu \mathrm{S} . \mathrm{cm}^{-1}$, valeur normale pour le Rhin à ce niveau et qui correspond à la limite entre des eaux moyennement riches et riches en électrolytes (KRAMMER et LANGE-BERTALOT, 1986).

Les valeurs de $\mathrm{pH}$ ont des extrêmes compris entre 5 et 8,3 , la majorité des mesures se situant dans une plage allant de 7,7 à 8,1 .

Les teneurs en azote et phosphore situent ces eaux aux limites inférieures de l'eutrophisation.

Il est important de noter qu'il n'y a pas, du point de vue de la physico-chimie de l'eau, de différences significatives pour ces paramètres entre les stations d'amont et d'aval.

\section{RÉSULTATS ET DISCUSSION}

Par rapport à la situation précédemment décrite (PIERRE, 1989a), la composition de la flore algale des stations ne révèle que des modifications mineures, essentiellement la présence d'espèces rarement (Ceratium hirundinella (O.F.M.) Schrank.) ou pas encore signalées, comme par exemple en station A : Hydrurus foetidus (Vill.) Trèv., Dinobryon divergens Imhof var. schauinslandii (Lemm.) Brunnth., Pleurotaenium trabecula (Ehr.) Naeg. ; en station B : Microcystis incerta (Lemm.) Lemm., Anthophysa vegetans (O.F.M.) Stein ; ou encore Celloniella sp., en station C. L'origine de ces espèces est à rechercher en amont, leur rareté correspondant à un dépôt accidentel.

De même, le peuplement diatomique se caractérise par sa stabilité (la nomenclature des Diatomées est établie suivant la "Sußwasserflora » de KRAMMER et LANGE-BERTALOT, 1986-1991). Sur plus de 300 taxons déjà signalés sur le site au cours de la période 1977-1987 (PIERRE, 1989b), seule une trentaine n'a pas été retrouvée. II s'agit dans tous les cas d'exemplaires isolés ou de quelques individus, dont l'origine allochtone est vraisemblable. II pouvait notamment s'agir d'entraînement depuis l'amont, en particulier depuis la région alpine, comme pour Achnanthes coarctata Bréb., Anomoeoneis vitrea (Grun.) Ross., Cocconeis neothumensis Krammer, Cymatopleura brunii Petit et Brun, Diploneis alpina Meister, Eunotia monodon Ehr., Frustulia rhomboides (Ehr.) de Toni var. saxonica (Rabh.) de Toni, Gomphonema ventricosum Gregory, Navicula angusta Grun., Neidium binodiforme Krammer, N. dilatatum (Ehr.) Cleve, Nitzschia sublinearis Hust., Pinnularia subcapitata Gregory ou P. sudetica (Hilse) Peragallo.

La minéralisation des eaux du Rhin est aussi suffisamment élevée pour expliquer l'observation d'autres espèces plus ou moins inféodées à ce facteur, l'apport étant envisageable depuis des localités en relation avec le gisement potassique d'Alsace et enrichies en sels. $\mathrm{Ce}$ pourrait être le cas d'Achnanthes clevei Grun. var. rostrata Hust., Gyrosigma scalproides (Rabh.) Cleve, Mastogloia smithii Thwaites var. lacustris Grun., Navicula digitoradiata (Greg.) Ralfs, N. halophila (Grun.) Cleve, N. protracta (Grun.) Cleve, Nitzschia levidensis (W. Sm.) Grun., N. tryblionella Hantzsch et Stauroneis prominula (Grun.) Hust. 
Enfin, parmi les espèces non retrouvées au cours des années' 1988=1995, figurent Actinocyclus ehrenbergii Ralfs et ses variétés crassus (W. Sm.) Hust et tenellus (Bréb.) Hust., Actinoptychus undulatus (Bail.) Ralfs, Caloneis ampliatum (Ehr.) Krammer, C. schumanniana (Grun.) Cleve var. biconstricta (Grun.) Reich., C. westii (W. Sm.) Hendey, Cocconeis disculus (Schumann) Cleve; Coscinodiscus marginatus Ehr., Cyclotella iris Brun, C. stelligera (Cleve et Grun.) v. H., Cyclotella stylorum Brightw., Navicula cohnii (Hilse) Lange-Bert., N. meniśculus Schumann, N. minuscula Grun. var. muralis Lange-Bert., N. striolata (Grün.) Lange-Bert., Neidium affine (Ehr.) Pfitzer, N. productum (W. Sm.) Cleve, Nitzschia amphibia Grun., N. nana Grun., N. sigma (Kütz.) W. Sm., Pinnularia gibba Ehr., P. globiceps Ehr., P. major (Kütz.) Rabh., Raphoneis amphiceros Ehr., Rhopalodia gibba (Ehr.) O. Müll., R. gibberula (Ehr.) O. Müll., ainsi que Stauroneis acuta W. Sm.

L'absence de ces espèces dans les relevés de la période 1988-1995 se trouve en grande partie compensée par la manifestation de Diatomées non encore recensées dans les stations, et dont l'origine allochtone est là encore probable sinon certaine. Il est, de la même façon, possible de distinguer des espèces devant leur origine à une dérive depuis la partie alpine du cours du Rhin, et d'autres pouvant trouver dans la minéralisation de l'eäu un facteur explicatif plus ou moins satisfaisant.

A la première catégorie, correspondent Amphora inariensis Krammer, Caloneis alpestris (Grun.) Cleve, Cyclotella bodanica Grun., C. comensis Grun., Cymbella gracilis (Ehr.) Kütz., C. lata Grun., Diatoma hyemalis (Roth) Heiberg, Eunotia formica Ehr., Fragilaria biceps (Kütz.) Lange-Bert., Nitzschia homburgiensis Lange-Bert., Pinnularia borealis Ėhr., Surirella spiralis Kütz.

Sont en accord avec la qualité de l'eau des stations: Cyclotella bodanica var. affinis (Grun.) Cleve-Euler, Denticula tenuis Grun., Diatoma moniliformis Kütz., Diploneis mauleri (Brun) Cleve, Navicula pseüdotuscula Hust., N. pseudanglica Lange-Bert., N. viridula (Kütz.) Ehr. var. rostellata (Kütz.) Cleve, N. vulpina Kütz., Pleurosira laevis (Ehr.) Compère, Surirella capronii Brébisson. II s'y ajoute trois espèces réputées d'eaux saumâtres, voire marines : Calonieis permagria (Bäiley) Cleve, Navicula crucicula (W. Sm.) Donkin, N. integra (W. Sm.) Ralfs, trouvées à l'état d'exemplaires isolés.

La diversité en espèces du peuplement diatomique des trois stations obéit, depuis les premières observations, à une hiérarchisation précise (Tableau I).

La station $C$ est habituellement la plus riche en nombre d'espèces, devant les stätions $B$ et $A$.

L'utilisation du paramètre " diversité en espèces " a été imposée par les conditions de récolte et la nature des prélèvements. Ainsi, la station $A$, en amont, est très souvent caractérisée par la dominance d'une seule espèce, Diatoma vulgaris Bory ou de son morphotype linearis, qui peuvent notamment au printemps représenter plus de $95 \%$ du peuplement, en rélation avec leur épiphytisme sur Cladophora glomerata (L.) Kütz., alors en pleine période de croissance. II s'y ajoute $2 \%$ pour Cocconeis pediculus Ehr. ou Rhoicosphenia abbreviata (Ag.) L.-B. selon la pläge observée, avec un complément de quelques espèces ișolées. Ainsi, à un comptage limité à quelques centaines d'individus correspondra une liste limitée à une dizaine d'espèces. Des comptages plus importants sont difficilement envisageables dans le cadre d'un suivi ; dans ces conditions, l'utilisation de cinq classes d'abondance n'ést pas irréaliste et permet de pousser le dépouillement de plusieurs préparations, soit 3 à $4 \mathrm{~cm}^{2}$, dè manière à obtenir une liste relativement complète bien que non exhaustive des taxons présents sur la station.

A l'inverse, la station d'aval $C$ est caractérisée par un envasement important qui induit une compétition au bénéfice de Vaucheria sp., supportant un recouvrement de Cocconeis pediculus, aux dépens de Cladophora glomerata qui se maintient difficilement et, de ce fait, au détriment de Diatoma vulgaris. Ces conditions se traduisent par une diversité dü peuplement, évidentè à l'examen microscopique.

Depuis l'origine des observations, donc dès avant la divergence du Centre de Production Nucléaire, la même méthodologie de prélèvements a opposé la faible diversité de la station 
d'amont liée à une ou deux espèces dominantes, à la diversité observée dans la station d'aval avec un partage beaucoup plus large des dominances. II semble difficile de quantifier et de comparer, par une même formule, deux stations dont l'une ne livre dans un champ microscopique qu'une seule espèce, alors que l'autre en révèle une dizaine.

Tableau I : Richesse spécifique des stations.

Table I : Species diversity of stations.

\begin{tabular}{|c|c|c|c|c|}
\hline Année & Mois & Station A & Station B & Station C \\
\hline \multirow[t]{4}{*}{1988} & 02 & 68 & 74 & 74 \\
\hline & 05 & 47 & 66 & 72 \\
\hline & 08 & 39 & 50 & 70 \\
\hline & 10 & 64 & 73 & 80 \\
\hline \multirow[t]{4}{*}{1989} & 02 & 42 & 59 & 55 \\
\hline & 05 & 33 & 57 & 57 \\
\hline & 08 & 59 & 67 & 74 \\
\hline & 10 & 39 & 64 & 64 \\
\hline \multirow[t]{4}{*}{1990} & 02 & 45 & 69 & 81 \\
\hline & 05 & 41 & 67 & 76 \\
\hline & 08 & 65 & 68 & 80 \\
\hline & - & - & - & - \\
\hline \multirow[t]{4}{*}{1991} & 02 & 47 & 62 & 61 \\
\hline & 05 & 53 & 66 & 73 \\
\hline & 08 & 55 & 78 & 85 \\
\hline & 10 & 52 & 72 & 73 \\
\hline \multirow[t]{4}{*}{1992} & 03 & 56 & 74 & 78 \\
\hline & 05 & 67 & 67 & 86 \\
\hline & 08 & 35 & 50 & 53 \\
\hline & 10 & 45 & 57 & 70 \\
\hline \multirow[t]{4}{*}{1993} & 02 & 47 & 52 & 52 \\
\hline & 05 & 45 & 59 & 59 \\
\hline & 08 & 52 & 53 & 75 \\
\hline & 10 & 62 & 75 & 86 \\
\hline \multirow[t]{4}{*}{1994} & 02 & 56 & 65 & 84 \\
\hline & 05 & 51 & 54 & 80 \\
\hline & 08 & 51 & 42 & 80 \\
\hline & 10 & 45 & 62 & 77 \\
\hline \multirow[t]{4}{*}{1995} & 02 & 49 & 54 & 72 \\
\hline & 05 & 59 & 64 & 73 \\
\hline & 08 & 34 & 43 & 52 \\
\hline & 10 & 36 & 40 & 69 \\
\hline
\end{tabular}


Sur la période 1988-1995, la station C est constamment la plus riche en espèces, devant les stations $B$ et $A$, ce qui conforte les résultats antérieurs. Une exception se manifeste en août 1994 où $A$ devance $B$ (51 espèces contre 42), ce que les conditions de prélèvements peuvent expliquer : le matériel issu de B était limité à quelques touffes de Bryophytes, sans raclage semble-t-il du support, ce qui a fortement réduit la richesse spécifique de la station. De telles inversions se manifestent de temps à autre et peuvent le plus souvent être rattachées à une particularité du prélèvement.

L'époque à laquelle se manifeste la diversité taxonomique la plus élevée ne paraît pas définie : c'est en février (1990 et 1994), en mai (1992 et 1995), en août (1989 et 1991) ou en octobre (1988 et 1993).

Enfin, ces trois stations sont situées à proximité les unes des autres et le fonctionnement du Centre de Production Nucléaire ne modifie pas la qualité de l'eau, à l'exception d'un très léger échauffement : les modifications qualitatives et quantitatives observées dans les stations ne peuvent pas être expliquées par une variation de la qualité de l'eau.

Si l'on recherche les espèces dominantes (occurrence supérieure ou égale à $25 \%$ du nombre d'individus, classe IV ou V), 12 taxons apparaissent : Cocconeis pediculus et C. placentula Ehr., Diatoma ehrenbergii Kütz., D. vulgaris, Gomphonema olivaceum (Horn.) Bréb., Melosira varians Agardh, Navicula lanceolata (Ag.) Ehr., N. nivalis Ehr., N. tripunctata (O.F. Müll.) Bory, N. veneta Kütz., Nitzschia dissipata (Kütz.) Grun. et Rhoicosphenia abbreviata. Navicula est mieux représentée dans la station $B$, le ralentissement du courant dans le bassin conduisant aux entrées des grilles les favorisant ; Cocconeis et Diatoma étant plus aptes à résister au courant caractéristique des stations $\mathrm{A}$ et $\mathrm{C}$.

En résumé, la station $A$ est dominée par Diatoma vulgaris qui trouve sur les.Cladophora un ancrage favorable, ainsi que Cocconeis dans une moindre mesure. Nitzschia dissipata et Rhoicosphenia abbreviata sont les accompagnatrices caractéristiques.

Diatoma et Cocconeis sont encore bien représentées station $\mathrm{B}$, lorsque le prélèvement renferme des touffes de Bryophytes leur servant de support. Le fort ralentissement du courant au niveau du point de récolte favorise le développement de plages de Navicula.

Courant et brassage de l'eau caractérisent la station $C$, ainsi qu'un envasement permanent. La dominance d'une seule espèce est plus rare, il y a souvent partage entre deux ou trois taxons parmi les Cocconeis, Diatoma et Navicula.

\section{CONCLUSION}

Le suivi algologique mené ces dernières années sur le site de Fessenheim confirme les observations effectuées depuis 1977 au niveau de la quantité relative et de la qualité de la flore algale et diatomique, en particulier.

Les variations observées au niveau des peuplements diatomiques ne sont pas la conséquence d'une variation de qualité de l'eau, compte tenu de la proximité des stations (quelques kilomètres) et de l'impact du Centre de Production Nucléaire, limité à une faible élévation de température (DT $\pm 1^{\circ} \mathrm{C}$ ). II s'agit ici d'une réponse au cycle saisonnier, renforcée par les caractéristiques physiques propres à chaque station.

Environ 10\% seulement des espèces observées dans la première période (1977-1987) n'ont pas été retrouvées les années suivantes, alors qu'un nombre voisin de taxons sont aṕparus, inédits pour ces stations. II s'agit toujours d'espèces isolées, de statut discutable, mises en évidence par le dépouillement poussé des prélèvements. Une douzaine d'espèces, constantes dans le temps, constituent l'élément dominant des stations.

Les observations réalisées depuis 1977 ont été conduites avec une méthodologie constante, permettant une confrontation, à partir de prélèvements imposés. Dans les conditions de ce travail, il n'apparaît pas de modifications significatives dans les quantités relatives ou la composition de la flore algale du Rhin canalisé au niveau du site de Fessenheim. Chacune des stations confirme sa personnalité et sa stabilité dans le temps. 


\section{REMERCIEMENTS} France.

Ce travail a été réalisé dans le cadre du suivi écologique du site initié par Electricité de

\section{BIBLIOGRAPHIE}

KRAMMER K., LANGE-BERTALOT H., 1986-1991. Sußwasserflora von Mitteleuropa. Bd 2/1-4, Bacillariophyceae. Gustav Fischer Verlag, Stuttgart.

PIERRE J.F., 1989a. Evolution décennale de la microbiocénose du Rhin au niveau du site électronucléaire de Fessenheim (Haut-Rhin). Crypto.-Algol., 10 (2), 117-128.

PIERRE J.F., 1989b. Inventaire du peuplement diatomique du Rhin canalisé (1977-1987). Bull. Acad. Soc. lorr. Sc., 28 (3), 87-105.

Nota : Une bibliographie spécifique a été publiée antérieurement (PIERRE, 1989b). 